\title{
BMJ Open Survival of mechanically ventilated ward patients and association with organisational factors: a multicentre prospective study
}

\author{
Wai-Tat Wong (D) , ${ }^{1}$ Anna Lee (D) , ${ }^{1}$ Charles David Gomersall (D) , ${ }^{1}$ Lam-hin Shek, ${ }^{2}$ \\ Alfred Chan, ${ }^{3}$ Sheung-on So, ${ }^{4}$ Kai-cheuk Sin, ${ }^{4}$ Wai-ming Tang, ${ }^{5}$ Maria Sinn, ${ }^{6}$ \\ Lowell Ling (D) ${ }^{1}$
}

To cite: Wong W-T, Lee A, Gomersall CD, et al. Survival of mechanically ventilated ward patients and association with organisational factors: a multicentre prospective study. BMJ Open 2021;11:e052462. doi:10.1136/ bmjopen-2021-052462

- Prepublication history and additional supplemental material for this paper are available online. To view these files, please visit the journal online (http://dx.doi.org/10.1136/ bmjopen-2021-052462).

Received 26 April 2021 Accepted 08 December 2021

Check for updates

(c) Author(s) (or their employer(s)) 2021. Re-use permitted under CC BY-NC. No commercial re-use. See rights and permissions. Published by BMJ.

For numbered affiliations see end of article.

Correspondence to

Dr Lowell Ling;

lowell.ling@cuhk.edu.hk

\section{ABSTRACT}

Objectives Determine 90-day mortality of mechanically ventilated ward patients outside the intensive care unit (ICU) and its association with organisational factors.

Design Multicentre prospective observational study of mechanically ventilated ward patients. Modified Poisson regression was used to assess association between nurse to patient ratio (NPR) and 90-day mortality, adjusted for designated medical team, Society of Critical Care Medicine (SCCM) triage priority and centre effect. NPR was divided into low (1:9.6 to 1:10), medium (1:6 to $1: 8)$ and high (1:2.6). Sensitivity analysis was conducted for pneumonia with or without acute respiratory distress syndrome (ARDS) to assess magnitude of association.

Setting 7 acute public hospitals in Hong Kong.

Participants All 485 mechanically ventilated patients in wards from participating hospitals between 18 January 2016 and 17 April 2016 were recruited. Three hundred patients were included after excluding patients with limitation of therapy within 24 hours of intubation.

Main outcomes 90-day mortality, Mortality Prediction Model III Standardised mortality ratio (MPMIII 0 SMR). Results 201 patients died within 90 days after intubation $(67.0 \%, 95 \% \mathrm{Cl} 61.5 \%$ to $72.1 \%)$, with MPMIII ${ }_{0}$ SMR $1.88,95 \% \mathrm{Cl} 1.63$ to 2.17. Compared with high NPR, medium and low NPRs were associated with higher risk of 90-day mortality (adjusted relative risk $\left(\mathrm{RR}_{\mathrm{adj}}\right) 1.84,95 \% \mathrm{Cl} 1.70$ to 1.99 and $1.64,95 \% \mathrm{Cl}$ 1.47 to 1.83 , respectively). For 114 patients with pneumonia with or without ARDS, low to medium NPR, too sick to benefit from ICU (SCCM priority 4b), no ICU consultation and designated medical team were associated with risk of 90-day mortality $\left(\mathrm{RR}_{\text {adj }} 1.49\right.$, $95 \% \mathrm{Cl} 1.40$ to $1.58 ; \mathrm{RR}_{\text {adj }} 1.60,95 \% \mathrm{Cl} 1.49$ to 1.72 ; $\mathrm{RR}_{\text {adj }} 1.34,95 \% \mathrm{Cl} 1.27$ to $1.40 ; \mathrm{RR}_{\text {adj }} 0.85,95 \% \mathrm{Cl}$ 0.78 to 0.93 , respectively).

Conclusion The 90-day mortality rates of mechanically ventilated ward patients were high. NPR was an independent predictor of survival for mechanically ventilated ward patients.

\section{Strengths and limitations of this study}

- First multicentre prospective study of ventilation outcomes in general wards.

- Analysis of organisational factors associated with survival including ward setting, medical team and nursing ratio.

- Limited by use of intensive care mortality prediction models on general ward patients to calculate standardised mortality ratio.

- Observational study does not demonstrate causal relationship between organisational factors and survival.

\section{INTRODUCTION}

Invasive mechanical ventilation (MV) is potentially life saving; however, it is a complex procedure, and in high resource settings, invasive MV outside the perioperative period is provided in intensive care unit (ICU).

However, ICU beds are limited in many parts of the world, and in disasters and epidemics, there is the risk of an undersupply even in high resource settings. ${ }^{12}$ As a result, provision of MV outside an established ICU may be considered. ${ }^{3-6}$ However, there are very limited data on the outcome of patients receiving invasive MV in this setting. Existing data from single-centre studies indicate a hospital mortality of $68 \%-89 \%$, but there is little data on the factors associated with mortality. ${ }^{3}{ }^{5-8} \mathrm{We}$ carried out a multicentred observational study to prospectively document the outcomes of patients who were ventilated in a non-ICU environment and determine the organisational factors, particularly nurse to patient ratios (NPRs), associated with mortality within 90 days of endotracheal intubation. 


\section{METHODS}

\section{Study design}

This study was a multicentre prospective observational study to evaluate the outcome of patients receiving invasive MV in general wards.

\section{Setting and participants}

The study period was from 18 January 2016 to 17 April 2016 in seven acute public hospitals in Hong Kong. The seven hospitals included one tertiary teaching hospital and six acute general district hospitals. Their capacity ranged from 523 to 1935 acute beds, and total bed capacity was 9338 . The total number of adult ICU beds was 106 across these seven hospitals. Overall, ICU bed capacity is relatively low in Hong Kong at 7.1 ICU beds per 100000 population. ${ }^{1}$ Therefore, ventilated patients are often cared for on general wards when they are not referred for or refused from ICU admissions.

Hospital coordinators identified all patients who were intubated and given mechanical ventilator support in general medical wards during the study period. Patients who have been admitted to the ICU or High Dependence Unit during their hospital stay were excluded from the study. Patients started on MV but had limitation of therapy within 24 hours of intubation were also excluded. The hospital coordinators collected clinical data of all recruited patients and followed them up for the outcomes until 90 days after intubation. The usual clinical team made all the clinical decisions on patient management without any additional intervention from the study.

\section{Variables}

Data were collected for the assessment of the Mortality Prediction Model III at admission (MPM III $)_{0}$ : coma status, heart rate, systolic blood pressure, chronic renal insufficiency, cirrhosis, malignancy, acute renal failure, cardiac arrhythmia, cerebrovascular incident, gastrointestinal bleeding, intracranial mass effect, age, cardiopulmonary resuscitation within 24 hours before admission, MV within 1 hour of admission, medical or unscheduled surgery admission and full resuscitation status. ${ }^{9}{ }^{10}$ For the purposes of this study, because the patients were not admitted to ICU, 'admission' time was defined as the time of intubation. Sequential organ failure assessment (SOFA) score within 24 hours of intubation was also collected. ${ }^{11}$ Society of Critical Care Medicine (SCCM) triage priority data were also retrieved from separate clinical audit on ICU consultations for patients whom have been assessed by the ICU team around the time of intubation. ${ }^{12}$ For the purpose of this study, we classified triage priority in the following groups: SCCM priority 1 (patients require intensive treatment and monitoring) or 2 (patients requiring intensive monitoring and potentially needing immediate intervention), SCCM priority 3 (patients have a reduced likelihood of recovery), priority 4a (patients too well to benefit from ICU), priority $4 \mathrm{~b}$ (patients too sick to benefit from ICU) and no ICU consultation. In public hospitals in Hong Kong, no ICU consultation usually implies that the chance of recovery is dismal and benefit from ICU care limited.

The reasons for invasive mechanical ventilator support were judged by the hospital coordinators, who enrolled the patient to the study, based on the information from the computerised hospital record, hard copy medical record and communications with the attending physicians. The reasons were categorised into eight groups: pneumonia with or without acute respiratory distress syndrome (ARDS), secondary ARDS, obstructive airway disease, acute pulmonary oedema, neurological disease, postcardiac arrest or others. The following data on the model of care while receiving ward MV were also collected: whether the patient was cared for in a designated ward, by a designated medical team of attending physicians, and the NPR. Designated ward is defined as a whole ward or cubicles inside a ward, which only admits patients requiring MV. Designated medical team is defined as a team of physicians who are routinely responsible for managing ventilated patients outside the ICU. Categories of NPR were determined retrospectively based on the ratios reported and were defined as low (1:9.6 to 1:10), medium (1:6 to 1:8) and high (1:2.6).

The primary endpoint of the study was mortality within 90 days of intubation. Twenty-eight days mortality and hospital mortality were also evaluated. Standardised mortality ratio (SMR) was calculated by dividing the observed hospital mortality by the predicted mortality using MPM III ${ }_{0}$.

Quality of ventilator care was evaluated by assessing the number of blood gas analysis, the rate of endotracheal tube events (obstruction or accidental extubation requiring reintubation), pneumothoraces during ward MV and the tidal volume per kg predicted body weight (PBW) by height on day 1 of MV.

\section{Statistical methods}

Sample size was determined on the basis of the desired precision of our estimate of mortality. Using nQuery Advisor V.7.0 (Statistical Solutions, Cork, Ireland) and the large sample normal approximation, a sample size of 246 patients was calculated, based on two-sided 95\% CI, which extended 0.05 from the observed proportion and an expected hospital mortality of $80 \%$ from a previous study comparing outcome of ventilated patients cared in ICU or on the ward. ${ }^{3}$

Discrete variables are expressed as counts (percentage), and continuous variables as mean and $\mathrm{SD}$ or median and IQR as appropriate after assessing normality visually and using the Shapiro-Wilk's test. Complete case analysis was used to describe the baseline distribution of SOFA, $\mathrm{P}_{2} \mathrm{O}_{2} / \mathrm{F}_{\mathrm{i}} \mathrm{O}_{2}$ ratio, tidal volume, arterial blood gases, ICU consultations and SCCM priority and in the regression models.

We compared NPR group characteristics using Kruskal-Wallis test with multiple pairwise comparisons adjusted with a Bonferroni correction. The SMR with 95\% CIs was estimated using Open Source 
Epidemiologic Statistics for Public Health (OpenEpi) V.3.01 software. ${ }^{13}$

The duration of follow-up was calculated from the start of MV on the ward to date of death in hospital or hospital discharge to home or another institution within 90 days. The association between NPR groups and risk of mortality within 90 days of commencing MV on the ward (relative risk (RR), 95\% CI) was assessed using a modified poisson regression model with robust SE estimation, adjusting for hospital centre effects, type of ward (not designated, designated), medical team (not designated, designated) and SCCM triage priority. ${ }^{14}$ These variables for statistical modelling were chosen using a directed acyclic graph approach in DAGitty software V.3.0 (online supplemental file 1). ${ }^{15}$ Age was not included in the regression model as it was not a confounder for the association between NPR and 90-day mortality. A modified poisson regression model with robust SE estimation was chosen after finding serious violations of the proportional hazard assumption in Cox survival analyses, and lack of adequate model fits using shared frailty parametric models. ${ }^{16}{ }^{17}$ In order to better inform the management of the current COVID-19 pandemic, a subgroup analysis of those patients with pneumonia (with or without ARDS) was carried out using the same methodology. Due to the smaller sample size in the subgroup analysis, low and medium NPR groups were combined. Data analyses were performed using
Stata V.16.1 (StataCorp). The level of significance was set at $\mathrm{p}<0.05$.

\section{Patient and public involvement}

Patients and the public were not involved in the design and conduct of this study.

\section{RESULTS}

\section{Whole cohort}

Of the 485 patients who were intubated and put on MV in the medical wards from 18 January 2016 to 17 April 2016, 185 were excluded due to limitation of support within 24 hours of intubation (figure 1). Across the seven hospitals, various combinations of different levels of NPR, designated wards and designated medical teams were adopted.

Baseline characteristics of the 300 included patients (table 1) showed that a substantial proportion was mechanically ventilated due to pneumonia with or without ARDS (38.3\%). Overall, the cohort had a 90-day mortality at $67.0 \%$ (95\% CI $61.5 \%$ to $72.1 \%$ ). Hospital mortality was $62.3 \%$ (95\% CI $56.7 \%$ to $67.7 \%$ ) with an SMR of 1.88 (95\% CI 1.63 to 2.17). Two hundred and sixty-seven patients $(91.7 \%)$ were categorised as priorities $3(52.2 \%)$ or $4 \mathrm{~b}(10.3 \%)$, or not evaluated for ICU admission $(29.2 \%)$, as benefit from ICU care was expected to be limited.

Baseline tidal volume and height were documented in $274(91.3 \%)$. Median (IQR) tidal volume was 7.6 (6.7 to 8.6$) \mathrm{mL} / \mathrm{kg}$. Other measures of ventilator care

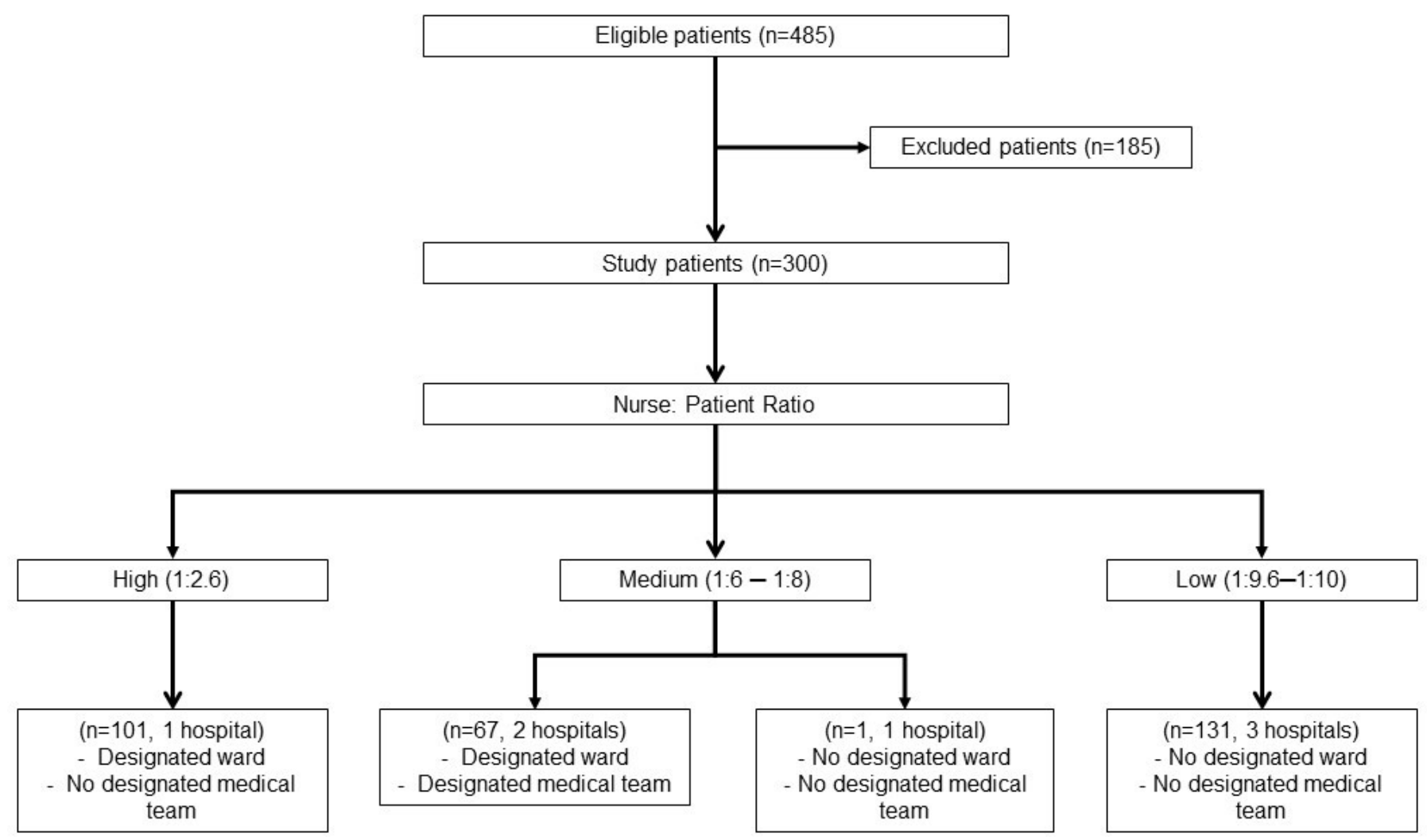

Figure 1 Patient flow diagram. 
Table 1 Baseline demographics

\begin{tabular}{|c|c|c|}
\hline Characteristics & All patients & Patients with pneumonia \pm ARDS \\
\hline Number of patients & 300 & 115 \\
\hline Median (IQR) age, year & $74.0(65.0-83.0)$ & $77(68-85)$ \\
\hline Male (\%) & $214(71.3)$ & $85(73.9)$ \\
\hline \multicolumn{3}{|l|}{ Reasons for requirement of MV support (\%) } \\
\hline Pneumonia without ARDS & $53(17.7)$ & \\
\hline Pneumonia with ARDS & $62(20.7)$ & \\
\hline Obstructive airway disease & $41(13.7)$ & \\
\hline Secondary lung injury & $5(1.7)$ & \\
\hline Neurological disease & $31(10.3)$ & \\
\hline Post cardiac arrest & $53(17.7)$ & \\
\hline Pulmonary oedema & $35(11.7)$ & \\
\hline Others & $20(6.7)$ & \\
\hline \multicolumn{3}{|l|}{ SCCM triage priority $(\%)^{\star}$} \\
\hline $\begin{array}{l}\text { Priority } 1 \text { (patients require intensive treatment and } \\
\text { monitoring) }\end{array}$ & $4(1.4)$ & $2(1.8)$ \\
\hline $\begin{array}{l}\text { Priority } 2 \text { (patients require intensive monitoring and } \\
\text { potentially need immediate intervention) }\end{array}$ & $11(3.8)$ & $3(2.6)$ \\
\hline Priority 3 (patients have a reduced likelihood of recovery) & $152(52.2)$ & $69(60.5)$ \\
\hline Priority 4a (patients are too well to benefit from ICU) & $9(3.1)$ & $2(1.8)$ \\
\hline Priority 4b (patients are too sick to benefit from ICU) & $30(10.3)$ & $11(9.6)$ \\
\hline No ICU consultation & $85(29.2)$ & $27(23.7)$ \\
\hline SOFA score (first 24 hours after intubation) & $9(8-11) \dagger$ & $9(8-10)^{\star \star}$ \\
\hline $\mathrm{PaO}_{2} / \mathrm{FiO}_{2}$ ratio $(\mathrm{mm} \mathrm{Hg})$ on day 0 & $208(104-410) \ddagger$ & $164(100-307) \dagger \dagger$ \\
\hline Duration of mechanical ventilation (hours) & $99(40-183)$ & $150(73-267)$ \\
\hline Tidal volume/ideal body weight on day 0 (ml/kg) & $7.6(6.7-8.6) \S$ & $7.7(7.0-8.6) \dagger \dagger$ \\
\hline Positive end-expiratory pressure $\left(\mathrm{cmH}_{2} \mathrm{O}\right)$ & $5(5-8) \emptyset$ & $6(5-8) \ddagger \ddagger$ \\
\hline Requiring vasopressor support (\%) on Day 0 & $172(57.3)$ & $68(59.1)$ \\
\hline Predicted number (\%) of in-hospital deaths by MPM III & $99.4(33.1)$ & $27.5(23.9)$ \\
\hline In-hospital deaths (\%) & $187(62.3)$ & $72(62.6)$ \\
\hline Deaths within 28 days of intubation (\%) & $185(61.7)$ & $69(60.0)$ \\
\hline Deaths within 90 days of intubation (\%) & $201(67.0)$ & $80(69.6)$ \\
\hline $\operatorname{SMR}(95 \% \mathrm{Cl})$ & $1.88(1.63-2.17)$ & $2.62(2.06-3.28)$ \\
\hline Hospital length of stay from intubation, days & $10(3-17)$ & $13(6-22)$ \\
\hline Hospital length of stay from hospital admission, days & $13(6-24)$ & $18(11-31)$ \\
\hline
\end{tabular}

Values are expressed as median and (IQR) unless specified.

*Missing data on SCCM triage priority in 9 of 300 patients.

$\dagger \mathrm{n}=290$.

$\neq \mathrm{n}=275$.

$\S \mathrm{n}=274$.

丹n $=296$.

** $n=109$.

$\dagger+n=107$.

$\ddagger \ddagger n=113$.

ARDS, acute respiratory distress syndrome; ICU, intensive care unit; MPM, Mortality Prediction Model; MV, mechanical ventilation; SCCM,

Society of Critical Care Medicine; SMR, standardised mortality ratio. 
Table 2 Observed and expected hospital mortality by reasons for requirement of mechanical ventilation support

\begin{tabular}{|c|c|c|c|c|}
\hline Reason & Number (\%) & Predicted mortality by MPM III $(\%)$ & Hospital mortality (\%) & SMR (95\% Cl) \\
\hline Pneumonia without ARDS & $53(17.7)$ & $13.5(25.4)$ & $31(58.5)$ & 2.30 (1.59 to 3.23$)$ \\
\hline Pneumonia with ARDS & $62(20.7)$ & $14.0(22.6)$ & $41(66.1)$ & 2.92 (2.12 to 3.93$)$ \\
\hline Secondary lung injury & $5(1.7)$ & $1.9(38.8)$ & $4(80.0)$ & 2.06 (0.66 to 4.98$)$ \\
\hline Neurological disease & 31 (10.3) & $11.0(35.5)$ & $18(58.1)$ & 1.64 (1.00 to 2.53$)$ \\
\hline Pulmonary oedema & $35(11.7)$ & $9.4(26.9)$ & $14(40.0)$ & 1.48 (0.84 to 2.43$)$ \\
\hline Others & $20(6.7)$ & $5.5(27.5)$ & $11(55.0)$ & $2.00(1.05$ to 3.47$)$ \\
\hline
\end{tabular}

.ARDS, acute respiratory distress syndrome; MPM III ${ }_{0}$, Mortality Prediction Model III at admission; SMR, standardised mortality ratio.

are shown in online supplemental file 2). All pairwise NPR groups comparisons were different from each for median number of arterial blood gas samples/ day done during MV (all $\mathrm{p}<0.015$ ). High NPR was significantly different to medium NPR and low NPR for number of ICU consultations done per day of MV ( $\mathrm{p}<0.001$ and $\mathrm{p}=0.002$, respectively). Low NPR was associated with more endotracheal tube events than high NPR $(\mathrm{p}=0.017)$ and medium NPR $(\mathrm{p}=0.004)$.

The SMRs varied by reason for MV (table 2) with the highest SMR in patients ventilated for pneumonia with ARDS. Compared with patients exposed to high NPR, patients exposed to medium and low NPRs were associated with a higher risk of mortality within 90 days (adjusted RR ( $\left.\mathrm{RR}_{\text {adj }}\right) 1.84,95 \%$ CI 1.70 to 1.99 and $1.64,95 \% \mathrm{CI} 1.47$ to 1.83 , respectively) after adjusting for hospital-centre effect, priority triage and designated medical team (table 3 ). Similar results were obtained after adjusting for hospital centre effect, priority triage and designated ward (online supplemental file 3 ).

\section{Pneumonia with or without ARDS subgroup}

Of the 115 patients, one patient did not have data on priority triage for the multivariable analysis. Compared with patients exposed to high NPR levels, patients exposed to medium to low NPRs were at higher risk of mortality within 90 days $\left(\mathrm{RR}_{\mathrm{adj}} 1.49\right.$, $95 \%$ CI 1.40 to 1.58 ) after adjusting for hospital centre effect, priority triage and designated medical

\begin{tabular}{|c|c|c|c|c|c|}
\hline Factor (n) & Mortality (n, \%) & RR $(95 \% \mathrm{Cl})$ & $P$ value & Adjusted RR $(95 \% \mathrm{Cl})$ & $P$ value \\
\hline \multicolumn{6}{|l|}{ Nursing: patient ratio* } \\
\hline High (101) & $48(47.5)$ & 1.00 & & 1.00 & \\
\hline Medium (68) & $56(82.4)$ & 1.73 (1.49 to 2.01$)$ & $<0.001$ & 1.84 (1.70 to 1.99$)$ & $<0.001$ \\
\hline Low (131) & $97(74.0)$ & 1.56 (1.36 to 1.78$)$ & & 1.64 (1.47 to 1.83$)$ & \\
\hline \multicolumn{6}{|l|}{ Medical team } \\
\hline Not designated (233) & $146(62.7)$ & 1.00 & & 1.00 & \\
\hline Designated (67) & $55(82.1)$ & 1.31 (0.97 to 1.77$)$ & 0.079 & 0.86 (0.75 to 0.99$)$ & 0.040 \\
\hline \multicolumn{6}{|l|}{ Ward } \\
\hline Not designated (132) & $98(74.2)$ & 1.00 & & & \\
\hline Designated (168) & $103(61.3)$ & 0.83 (0.54 to 1.27$)$ & 0.385 & Dropped from model du & to co-linearity \\
\hline \multicolumn{6}{|l|}{ Priority triage (\%) } \\
\hline SCCM priority 3 (152) & $101(66.4)$ & 1.00 & & 1.00 & \\
\hline $\begin{array}{l}\text { SCCM priority } 1 \text { or } 2 \\
\text { (15) }\end{array}$ & $7(46.7)$ & 0.70 (0.43 to 1.15$)$ & & 0.75 (0.50 to 1.14$)$ & \\
\hline SCCM priority 4a (9) & $2(22.2)$ & 0.33 (0.05 to 2.19$)$ & $<0.001$ & 0.41 (0.07 to 2.33 ) & $<0.001$ \\
\hline SCCM priority 4b (30) & $24(80.0)$ & 1.20 (1.06 to 1.37$)$ & & 1.24 (1.07 to 1.45$)$ & \\
\hline No ICU consultation (85) & $61(71.8)$ & 1.08 (0.86 to 1.36$)$ & & $1.21(1.00$ to 1.47$)$ & \\
\hline
\end{tabular}

$P$ values are for overall variable effect.

${ }^{*}$ High defined as one nurse: 2.6 patients. Medium defined as one nurse: 6-8 patients. Low defined as one nurse: 9.6-10 patients.

ICU, intensive care unit; RR, relative risk; SCCM, Society of Critical Care Medicine. 
Table 4 Association between various factors and risk of mortality within 90 days in 115 pneumonia with or without acute respiratory distress syndrome patients (unadjusted and adjusted RR)

\begin{tabular}{|c|c|c|c|c|c|}
\hline Factor (n) & Mortality (n, \%) & RR (95\% Cl) & $P$ value & Adjusted RR (95\% Cl) & $P$ value \\
\hline High (40) & $24(60.0)$ & 1.00 & & 1.00 & \\
\hline \multicolumn{6}{|l|}{ Medical team } \\
\hline Not designated (90) & $61(67.8)$ & 1.00 & & 1.00 & \\
\hline \multicolumn{6}{|l|}{ Ward } \\
\hline Not designated (50) & $37(74.0)$ & 1.00 & & & \\
\hline Designated (65) & $43(66.2)$ & 0.89 (0.76 to 1.06$)$ & 0.186 & Dropped from model du & to collinearity \\
\hline \multicolumn{6}{|l|}{ Priority triage (\%) } \\
\hline SCCM priority 4b (11) & $10(90.9)$ & 1.39 (1.27 to 1.53$)$ & & 1.60 (1.49 to 1.72$)$ & \\
\hline No ICU consultation (27) & $20(74.1)$ & 1.14 (0.96 to 1.34$)$ & & 1.34 (1.27 to 1.40$)$ & \\
\hline
\end{tabular}

$P$ values are for overall variable effect.

*High defined as one nurse: 2.6 patients. Medium defined as one nurse: 6-8 patients. Low defined as one nurse: 9.6-10 patients. $\mathrm{ICU}$, intensive care unit; RR, relative risk; SMR, standardised mortality ratio.

team (table 4). Similar results were obtained after adjusting for hospital centre effect, priority triage and designated ward (online supplemental file 4).

\section{DISCUSSION}

The 90-day mortality risk of patients who received MV in general wards was high $(67.0 \%)$ and hospital mortality was 1.88 times higher than would have been expected, based on severity of illness and admission to an average performance ICU. The difference between actual and expected mortality was greatest among those ventilated for pneumonia and ARDS, in whom the SMR (95\% CI) was 2.92 (2.12 to 3.93). NPR was an independent predictor of survival in the entire cohort (table 3) and in the subgroup ventilated for pneumonia with or without ARDS (table 4).

These data suggest that, in selected critically ill patients who were not admitted to ICU, MV outside an intensive care setting, although associated with a high mortality, may result in a substantial number of survivors. However, survival is strongly associated with nurse:patient ratio, SCCM priority and either designated ward or medical team. Our mortality was similar to that reported by two studies from Israel and one from Thailand but was substantially lower than previously reported from a single centre in Hong Kong. ${ }^{3-7}$ The association between NPR and survival is in keeping with previous data demonstrating an association between nurse:patient or nurse:nursing workload and mortality in critically ill patients. ${ }^{18-25}$ Our results are also consistent with a previous study showing higher workload, and reduced nurse staffing was associated with increased mortality in general wards. ${ }^{26}$ Furthermore, there were significant differences in frequency of arterial blood gas sampling and number of endotracheal tube events with different levels of nurse staffing.

Compliance with lung protective ventilation with a median tidal volume of 7.7 (7.0 to 8.6 ) $\mathrm{mL} / \mathrm{kg}$ PBW was comparable to the ventilation practice for non-ARDS patients in ICUs from high-income and middle-income countries of 7.9 (6.8 to 9.1 ) and 7.8 (6.8 to 9.1$) \mathrm{mL} / \mathrm{kg}$ PBW, respectively. ${ }^{27} 28$ Similarly, for subgroup of patients with ARDS, lung protective strategies adopted in our cohort were similar to the practice found in the LUNG SAVE study, which showed a mean $(95 \% \mathrm{CI})$ tidal volume of 7.6 (7.5 to 7.7$) \mathrm{mL} / \mathrm{kg} \mathrm{PBW.}{ }^{29}$ Our data suggest that compliance with lung protective strategy was generally good. However, a large portion of mechanically ventilated ward patients may still benefit from use of lung protective strategies.

The data related to the subgroup of patients with pneumonia with or without ARDS may be particularly relevant to the current pandemic of COVID-19, although it has been suggested that phenotypic expression of COVID-19 is different to other causes of primary ARDS. ${ }^{30}$ Guidelines on expansion of ICUs in a disaster recommend that ICU capacity be increased, if necessary, by up to three times. ${ }^{31-33}$ Our data show that there is low but substantial survival in these patients when invasively ventilated in general wards and provide support for the proposed ICU 
expansion, which is similar to ventilator care in non-ICU environment.

While the survival of mechanically ventilated ward patients may be promising, we also showed that survival is strongly associated with high NPR and care provided by designed medical team. LUNG SAFE study, an international multicentre study investigating the management of ARDS patients in ICU, found association between high NPR/physician-patient ratio and recognition of ARDS, which was associated with the use of lower tidal volume in ventilation. ${ }^{29}$ In the healthcare setting of COVID-19 pandemic, staffing levels for ventilated patients have been severely reduced, from a normal of one nurse per patient to one nurse for six patients, corresponding to our classification of medium NPR. ${ }^{34}$ Combining findings from our study and the LUNG SAFE study, any gain resulting from expansion of ICU facilities may be offset by a lower survival rate associated with inadequate staffing levels. Optimising the healthcare workers to patient ratio should be seriously considered in providing ventilator care in non-ICU environment or expanding ICU capacity.

The strengths of our study are that it is the first multicentre study of ventilation in general wards and the first to attempt to identify NPRs associated with survival. However, our data must be interpreted with caution. First, this was a purely observational study and, therefore, should not be interpreted as showing a causal relationship between NPR and survival. Furthermore, although we controlled for organisational characteristics (designated ventilation ward and medical team), it is possible that the demonstrated association between NPR and survival is due to unmeasured confounders. In addition, we are unable to exclude the possibility that the apparently superior survival in patients cared for with a high NPR is simply due superior care in one hospital. However, we believe this is unlikely given the evidence demonstrating an association between nurse:patient or nurse:nursing workload and mortality in critically ill patients. ${ }^{18-21}$ Moreover, systemic differences in organisation factors between hospitals are unlikely since all public hospitals are managed by under a single organisation. ${ }^{23} 24$

In addition, the high SMRs should not be interpreted as demonstrating that ward ventilation results in poor outcomes as the finding is confounded by the fact that most patients were refused admission to ICU. Since MPM $\mathrm{III}_{0}$ was designed to evaluate the severity of illness of patients admitted to ICU it may not be well calibrated for patients who are refused ICU admission. Furthermore use of time of intubation as definition of "admission" time for calculation of MPM III ${ }_{0}$ may have increased the estimated mortality. Calculation of estimated mortality includes a contribution from intubation within 1 hour of admission, by defining admission time as the time of intubation, we ensured that all patients met these criteria. An overestimate of expected mortality would have the effect of decreasing standard mortality ratios. Confounding by indication may also bias our cohort, although we have adjusted for each patient's SCCM triage priority. We were also unable to determine the reasons why some of the patients were not referred to ICU for admission, though not referring to ICU usually implies dismal chance of recovery and limited benefit from ICU care. In addition, the absolute mortality in our patients may not be applicable to other settings. As more than $70 \%$ of the cohort were evaluated by the ICU team and categorised as SCCM triage priority of $1-3$, and the ICU bed provision is very low in Hong Kong (2.5 per 100000 residents), a significant proportion of our cohort may be admitted to ICU for MV in other healthcare systems with higher ICU provision. ${ }^{1}$ However, in the current pandemic, the supply:demand imbalance in high ICU resource healthcare systems may be more similar to our study.

\section{CONCLUSIONS}

The 90-day mortality rates of mechanically ventilated ward patients were high. The difference between actual and expected mortality was greatest among those ventilated for pneumonia and ARDS. Ventilation practice with lung protective strategy in ward patients was comparable to recent practice in the critical care setting. Organisation of care including optimising NPR and assigning a designated team can improve survival for mechanically ventilated patients in non-ICU environment.

\section{Author affiliations}

${ }^{1}$ Anaesthesia and Intensive Care, The Chinese University of Hong Kong, Hong Kong SAR, China

${ }^{2}$ Department of Medicine and Geriatrics, Caritas Medical Center, Hong Kong SAR, China

${ }^{3}$ Department of Anaesthesia and Intensive Care, Tuen Mun Hospital, Hong Kong SAR, China

${ }^{4}$ Department of Intensive Care, Queen Elizabeth Hospital, Hong Kong SAR, China ${ }^{5}$ Department of Intensive Care, Princess Margaret Hospital, Hong Kong SAR, China ${ }^{6}$ Department of Medicine, Tseung Kwan 0 Hospital, Hong Kong SAR, China

\section{Twitter Anna Lee @AnnaLee_AIC and Lowell Ling @lingling7}

Acknowledgements We thank Ms. Jenny Ng and other nurses for their help with this study.

Contributors CDG and WTW designed the study. WTW, LL, LS, AC, SS, KS, WT and MS recruited patients and collected the data. WTW and AL analysed the data. WTW, $\mathrm{LL}$ and $C D G$ wrote the manuscript and other authors all revised and approved the manuscript. WTW is the guarantor.

Funding WTW was supported by the Young Investigator Research Grant 2015 from the Hong Kong College of Physicians. Funding number: N/A.

Competing interests None declared.

Patient consent for publication Not applicable.

Ethics approval This study involves human participants and was approved by the Joint Chinese University of Hong Kong-New Territories East Cluster Clinical Research Ethics Committee (2015.482), Kowloon West Cluster Research Ethics Committee (WH/EX-15-194(93-05)), Kowloon Central Cluster/Kowloon East Cluster Clinical Research Ethics Committee (KC/KE-15-0157/ER-1) and New Territories West Cluster Clinical and Research Ethics Committee (NTWC/CREC/15110) with waiver of informed consent.

Provenance and peer review Not commissioned; externally peer reviewed.

Data availability statement Data are available upon reasonable request. The datasets analysed during the current study are available from the corresponding author on reasonable request and approval from each participating site's ethics committee. 
Supplemental material This content has been supplied by the author(s). It has not been vetted by BMJ Publishing Group Limited (BMJ) and may not have been peer-reviewed. Any opinions or recommendations discussed are solely those of the author(s) and are not endorsed by BMJ. BMJ disclaims all liability and responsibility arising from any reliance placed on the content. Where the content includes any translated material, BMJ does not warrant the accuracy and reliability of the translations (including but not limited to local regulations, clinical guidelines, terminology, drug names and drug dosages), and is not responsible for any error and/or omissions arising from translation and adaptation or otherwise.

Open access This is an open access article distributed in accordance with the Creative Commons Attribution Non Commercial (CC BY-NC 4.0) license, which permits others to distribute, remix, adapt, build upon this work non-commercially, and license their derivative works on different terms, provided the original work is properly cited, appropriate credit is given, any changes made indicated, and the use is non-commercial. See: http://creativecommons.org/licenses/by-nc/4.0/.

\section{ORCID iDs}

Wai-Tat Wong http://orcid.org/0000-0002-7228-9486

Anna Lee http://orcid.org/0000-0003-2864-0045

Charles David Gomersall http://orcid.org/0000-0002-8494-8063

Lowell Ling http://orcid.org/0000-0001-6639-7344

\section{REFERENCES}

1 Phua J, Faruq MO, Kulkarni AP, et al. Critical care bed capacity in Asian countries and regions. Crit Care Med 2020;48:654-62.

2 Rhodes A, Ferdinande P, Flaatten $\mathrm{H}$, et al. The variability of critical care bed numbers in Europe. Intensive Care Med 2012;38:1647-53.

3 Hersch M, Sonnenblick M, Karlic A, et al. Mechanical ventilation of patients hospitalized in medical wards vs the intensive care unit--an observational, comparative study. J Crit Care 2007;22:13-17.

4 Kamio T, Masamune K. Mechanical ventilation-related safety incidents in general care wards and ICU settings. Respir Care 2018;63:1246-52.

5 Tang WM, Tong CK, Yu WC, et al. Outcome of adult critically ill patients mechanically ventilated on general medical wards. Hong Kong Med J 2012;18:284-90.

6 Wongsurakiat P, Sangsa N, Tangaroonsanti A. Mechanical ventilation of patients hospitalized on general medical ward: outcomes and prognostic factors. J Med Assoc Thai 2016;99:772-6.

7 Lieberman D, Nachshon L, Miloslavsky O, et al. Elderly patients undergoing mechanical ventilation in and out of intensive care units: a comparative, prospective study of 579 ventilations. Crit Care 2010;14:R48

8 Hersch M, Izbicki G, Dahan D, et al. Predictors of mortality of mechanically ventilated patients in internal medicine wards. J Crit Care 2012;27:694-701.

9 Higgins TL, Teres D, Copes WS, et al. Assessing contemporary intensive care unit outcome: an updated mortality probability admission model (MPM0-III). Crit Care Med 2007;35:827-35.

10 Higgins TL, Kramer AA, Nathanson $\mathrm{BH}$, et al. Prospective validation of the intensive care unit admission mortality probability model (MPM0-III). Crit Care Med 2009;37:1619-23.

11 Vincent JL, de Mendonça A, Cantraine F, et al. Use of the SOFA score to assess the incidence of organ dysfunction/failure in intensive care units: results of a multicenter, prospective study. Working group on "sepsis-related problems" of the European Society of Intensive Care Medicine. Crit Care Med 1998;26:1793-800.

12 Guidelines for intensive care unit admission, discharge, and triage. Task force of the American College of Critical Care Medicine, Society of Critical Care Medicine. Crit Care Med 1999;27:633-8.

13 OpenEpi. Open Source Epidemiologic Statistics for Public Health [program]. 3.01 version, 2013.
14 Zou G. A modified poisson regression approach to prospective studies with binary data. Am J Epidemiol 2004;159:702-6.

15 Attia JR, Oldmeadow C, Holliday EG, et al. Deconfounding confounding part 2: using directed acyclic graphs (DAGs). Med $J$ Aust 2017;206:480-3.

16 Katz MH. Multivariable analysis (a practical guide for clinicians and public health researchers. 3 ed. Cambridge University Press, 2011.

17 Kleinbaum DGK. Mitchel survival analysis: a Self-Learning text. 3 ed. Springer, 2012.

18 Lee A, Cheung YSL, Joynt GM, et al. Are high nurse workload/ staffing ratios associated with decreased survival in critically ill patients? a cohort study. Ann Intensive Care 2017;7:46.

19 Kane RL, Shamliyan TA, Mueller C, et al. The association of registered nurse staffing levels and patient outcomes: systematic review and meta-analysis. Med Care 2007;45:1195-204.

20 Stone PW, Mooney-Kane C, Larson EL, et al. Nurse working conditions and patient safety outcomes. Med Care 2007;45:571-8.

21 Cho S-H, Hwang JH, Kim J. Nurse staffing and patient mortality in intensive care units. Nurs Res 2008;57:322-30.

22 West E, Barron DN, Harrison D, et al. Nurse staffing, medical staffing and mortality in intensive care: an observational study. Int J Nurs Stud 2014;51:781-94.

23 Sakr Y, Moreira CL, Rhodes A, et al. The impact of hospital and ICU organizational factors on outcome in critically ill patients: results from the extended prevalence of infection in intensive care study. Crit Care Med 2015;43:519-26.

24 Checkley W, Martin GS, Brown SM, et al. Structure, process, and annual ICU mortality across 69 centers: United States Critical Illness and Injury Trials Group Critical IIIness Outcomes Study. Crit Care Med 2014;42:344-56.

25 Neuraz A, Guérin C, Payet C, et al. Patient mortality is associated with staff resources and workload in the ICU: a multicenter observational study. Crit Care Med 2015;43:1587-94.

26 Griffiths P, Maruotti A, Recio Saucedo A, et al. Nurse staffing, nursing assistants and hospital mortality: retrospective longitudinal cohort study. BMJ Qual Saf 2019;28:609-17.

27 Neto AS, Barbas CSV, Simonis FD, et al. Epidemiological characteristics, practice of ventilation, and clinical outcome in patients at risk of acute respiratory distress syndrome in intensive care units from 16 countries (PRoVENT): an international, multicentre, prospective study. Lancet Respir Med 2016;4:882-93.

28 Pisani L, Algera AG, Serpa Neto A, et al. Epidemiological characteristics, ventilator management, and clinical outcome in patients receiving invasive ventilation in intensive care units from 10 Asian middle-income countries (PRoVENT-iMiC): an international, multicenter, prospective study. Am J Trop Med Hyg 2021.

29 Bellani G, Laffey JG, Pham T, et al. Epidemiology, patterns of care, and mortality for patients with acute respiratory distress syndrome in intensive care units in 50 countries. JAMA 2016;315:788-800.

30 Gattinoni L, Chiumello D, Caironi P, et al. COVID-19 pneumonia: different respiratory treatments for different phenotypes? Intensive Care Med 2020;46:1099-102.

31 Hick JL, Einav S, Hanfling D, et al. Surge capacity principles: care of the critically ill and injured during pandemics and disasters: chest consensus statement. Chest 2014;146:e1S-16.

32 Sprung CL, Zimmerman JL, Christian MD, et al. Recommendations for intensive care unit and hospital preparations for an influenza epidemic or mass disaster: summary report of the European Society of intensive care medicine's task force for intensive care unit triage during an influenza epidemic or mass disaster. Intensive Care Med 2010;36:428-43.

33 Gomersall CD, Joynt GM, Ho OM, et al. Transmission of SARS to healthcare workers. The experience of a Hong Kong ICU. Intensive Care Med 2006;32:564-9.

34 Dunhill L. Exclusive: intensive care staffing ratios dramatically diluted. Health Service J 2020. 